\title{
Magnetic source images of human brain functions
}

\author{
N. G. GENÇER and S. J. WILLIAMSON \\ New York University, New York, New York
}

\begin{abstract}
The advent of large arrays of superconducting magnetic field sensors makes it possible to properly sample the topography of the magnetic field pattern across the human scalp with a temporal resolution of a few milliseconds. These capabilities can be exploited for computing a best estimate of the spatiotemporal evolution of electrical currents within coherently active neural populations distributed across the cerebral cortex. Data from 200 sensors can be interpreted by the method of singular value decomposition to compute a best estimate for the strengths of more than 9,000 current elements that define the magnetic source image moment by moment. Recently, an extension of this technique has been developed to characterize the cortical sources of alpha rhythm. This holds promise for providing a means of identifying the cortical regions that participate in cognitive functions such as mental imagery.
\end{abstract}

Advances in technology during the past 20 years have provided a variety of methods for imaging the functional organization of the human brain. One of the earliest is positron emission tomography (PET), which identifies where radioactive labels accumulate as a consequence of local biochemical processes. More recently, improvements in the sensitivity of magnetic resonance imaging (MRI) have provided a means of monitoring local changes in blood flow rate and blood volume, which are exploited by the technique of functional magnetic resonance imaging (fMRI). Within the last 3 years, large arrays of superconducting sensors have become available that can detect the very weak magnetic fields of local populations of coherently active neurons within the brain, with a temporal resolution of a few milliseconds. The dominant source of these magnetic fields is the intracellular current of pyramidal cells, which are oriented perpendicular to the surface of the cerebral cortex. These magnetic field measurements can be exploited to compute the spatiotemporal evolution of electrical currents across the cerebral cortex. Such a representation is called a magnetic source image (MSI).

To properly represent the pattern of transcortical current across the entire cerebral cortex requires an array of several thousand current elements, each oriented perpendicular to the local surface of the cortex, as illustrated in Figure 1. The present representation is composed of 9,104 current elements. These are also called "current dipoles" because at one end of the element they emit current into the surrounding medium and at the other end they take in current from the medium. They may be augmented by additional current dipoles located within subcortical regions where neural activity is expected, based

This work was supported in part by a grant to New York University from the Horace W. Goldstein Foundation. Correspondence should be addressed to S. J. Williamson, Department of Physics, 2 Washington Place, New York University, New York, NY 10003-6211 (e-mail: sjwmson@) nyu.edu). on the geometry of the individual's brain as defined by high-resolution MRIs.

Figure 1 shows the array of 234 magnetic field sensors across the spherical scalp, with neighboring sensors separated by a distance of approximately $2 \mathrm{~cm}$. The cortical surface in this figure was obtained from an MRI of a subject by using the Wrapper algorithm (Guéziec \& Dean, 1994). This extracts the surface of interest using triangular elements with an approximately constant density of triangles. The surface normals at the vertices are also calculated to define the directions of the image dipoles. In this study, the extracted surface includes some part of the spinal cord and originally had 26,862 triangles with 13,119 vertices. However, in order to constrain the problem to dipoles located within a reasonable distance from the scalp, deep sources of the brain stem and portions of the cortex within $6 \mathrm{~cm}$ of the center of the spherical head are not considered as active. This region is shown by dashed lines in Figure 1. Consequently, a total of 9,104 current dipoles are uniformly distributed across the cortical surface, and they are used for constructing the forward problem for the data provided by the sensors.

The distribution of current across these elements comprises the MSI. For practical reasons, the number of magnetic field sensors that can be used to sample the magnetic field pattern is limited to 101 . Consequently, the number of unknowns greatly exceeds the number of values provided by the measurements. The sensors are spaced at approximately $2-\mathrm{cm}$ intervals across the spherical surface of the scalp (Figure 1). Each is positioned $9.5 \mathrm{~cm}$ from the center of the head to measure the radial component of the magnetic field. The array covers the upper surface of the spherical head but does not quite extend across the lower portion of the sphere, where neck and mouth structures would complicate the geometry of a truly realistic head. Data from such a large array of sensors can be interpreted by the method of singular value decomposition (SVD) to compute a "best estimate" for the distribution of strengths of the current dipoles. 


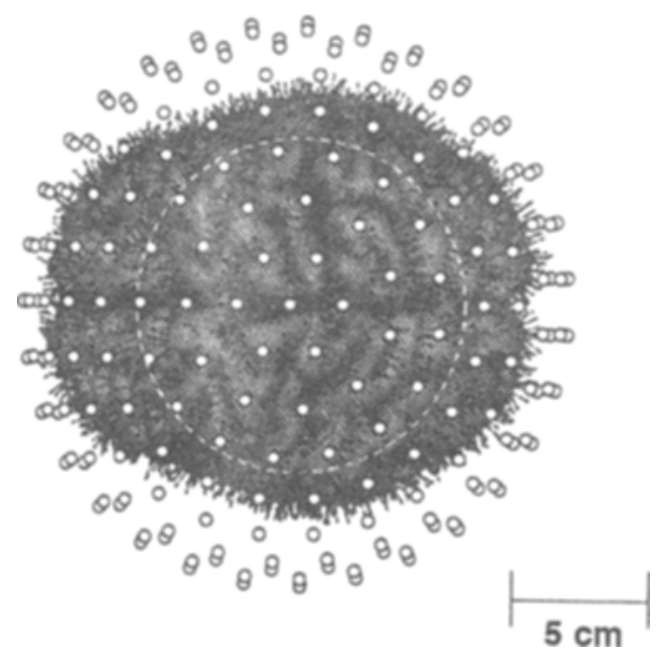

Figure 1. Array of 9,104 current elements distributed across the convoluted surface of a realistic cerebral cortex, viewed from over the vertex. No sources are allowed within the region of the brain stem, represented here by a sphere of 6-cm radius from the center of the head as defined by dashed lines. Locations of magnetic sensors are also depicted.

To illustrate the procedure for computing an MSI, the model head is represented by a realistic cortex surrounded by a three-layer conducting sphere representing the cerebrospinal fluid, skull, and scalp, with the scalp being $8.8 \mathrm{~cm}$ from the center of the head. Because the magnetic field pattern outside a head having spherical symmetry is independent of the radial variation of electric conductivity, the head can be viewed as having a uniform conductivity. Consequently, the magnetic field pattern outside the head is determined only by the distance of the neural source from the center of the sphere. By contrast, the pattern of electrical potential across the surface of the spherical head is sensitive to the radial dependence in electrical conductivity.

For a given source geometry, a linear system of equations $\mathbf{b}=\mathbf{L} \mathbf{q}$ relates the set of measurements obtained by an array of magnetic field sensors at a moment in time, represented by the components of the vector $b$, to the distribution of strengths of the current dipoles, represented by the components of the vector $q$ (George, Lewis, Ranken, Kaplan, \& Wood, 1991; Wang, Williamson, \& Kaufman, 1992). The matrix $\mathbf{L}$ is called the "lead-field matrix," and each element $L_{i j}$ represents the contribution of the $j$ th unit current dipole to the $i$ th sensor's output. To achieve an image of high resolution, the dipoles are closely spaced. Typically, this means several thousand dipoles must be distributed across the cerebral cortex. Because the number of sensors is on the order of hundreds, the number of measurements is much less than the number of unknowns (the dipole moments); consequently, there is no unique solution for the inverse problem.

Linear inversion methods have been applied by many investigators to interpret magnetic field data (or scalp voltage data) of this type (Dale \& Sereno, 1993; George et al., 1991; Geselowitz \& Miller, 1973; Hughett, 1995; Jeffs, Leahy, \& Singh, 1987; Ramon, Meyer, Nelson, Spelman, \& Lamping, 1993; Wang, Kaufman, \& Williamson, 1993; Wang et al., 1992), and an overview of the relationships between different forms of inverse solutions has been given by Hughett (1995). Among them, the minimumnorm least-squares (MNLS) inverse has been emphasized for locating neuronal activity with magnetic data. This is attained by employing a method based on the Moore-Penrose pseudoinverse (Albert, 1972; Moore, 1920; Penrose, 1955). The MNLS inverse provides a best estimate in the sense of minimizing the square of the error between the predicted field pattern and the data, while also achieving the most economical solution by minimizing the total strength (norm) of the image among all such solutions. This latter feature suppresses any current configurations that produce no detectible magnetic field. The MNLS image provides a quantitatively accurate characterization for the source strength and reasonable spatial resolution if the measurements are noise free (Gençer \& Williamson, in press). However, in the presence of noise, this inverse attempts to account for the noise as well, and depending on the ill-posedness of the inverse problem, the deterioration of the quality of the MNLS solutions may become unacceptable (Hughett, 1995; Jackson, 1972). An example is provided in Figure 2.

\section{CORTICAL BASIS FUNCTIONS}

The spatial distribution of neural activity provided by the MNLS inverse can be represented by a set of activity patterns (basis functions) that extend across the entire cortex. Each basis function specifies a pattern of current dipoles that is directed into the cortex in some regions and outward in others, and its image strength is normalized so that the total current dipole power (sum of squares of dipoles) is unity. Moreover, these basis functions are orthogonal to each other, meaning that the product of the values of the current dipole moments associated with any two basis functions, when summed over the entire surface of the cortex, is zero.

The MNLS inverse for a measured potential pattern on the scalp is the sum of the contributions from all of the basis functions, each weighted by a coefficient that is obtained by the Moore-Penrose method using singular value decomposition (SVD; Golub \& Van Loan, 1989). Some of the basis functions are strongly represented in the measurements, while others are weaker than the noise level. The spatial configurations of the basis functions are determined by the geometry of the sensor array and the geometry of the image space, which in the present case of interest is the convoluted surface of the cerebral cortex. It is convenient to order the basis functions according to decreasing total power, so that gross spatial features of the cortical activity are represented by functions of low index number, and finer features, by the weaker functions 
of high index number. The sensitivity of the image to measurement noise for a specific montage of sensors depends on how rapidly the strengths of the basis functions decrease with increasing index number. This is called the "singular value decay curve." If the singular values slowly decrease, many more functions of high-index number are above the noise, and consequently the image will display finer features than otherwise.

Basis functions having strengths that are in the range of the noise level limit the image quality, and the image may exhibit instabilities (Shim \& Cho, 1981). One approach to avoid these problems is to eliminate the basis functions whose signals are comparable to the noise level. This method is known as the "truncated SVD pseudoinversion," and the truncation criteria can be based on the signal-to-noise ratio $(\mathrm{S} / \mathrm{N})$ of the measurements (Gençer \& Williamson, in press; Jeffs et al., 1987). Unfortunately, the loss of high-index basis functions degrades the details of the image.

\section{FORWARD PROBLEM}

As mentioned earlier, the relationship between the dipole moments of the current dipoles and the magnetic field measurements near the scalp can be expressed as a system of linear equations. The general solution for the fields can be written as a linear combination of associated Legendre polynomials. The set of linear equations relating 9,104 current dipole moments to 234 magnetic field sensors can then be represented by the following:

$$
\mathbf{b}=\mathbf{L q},
$$

where $b$ represents the $234 \times 1$ vector of field measurements, and $\mathbf{L}$ is the $234 \times 9,104$ lead field matrix.

The SVD method identifies the basis functions that comprise the image and their respective field patterns at the scalp. The relative strengths of these patterns are specified by their singular values. By examining a basis function's pattern of strength across the cerebal cortex, it is possible to gain insight on which areas of the brain it emphasizes. Gençer and Williamson (in press) have provided detailed examples of these functions, as well as those that characterize the information yielded by measurements of voltages across the scalp (EEG). The singular values of the series of basis functions for magnetic data decay almost exponentially with their position in the sequence, as illustrated in Figure 3.

\section{INVERSE PROBLEM}

An example of the images obtained from simulated magnetic data is provided in Figure 2. The neural activity lies on the right wall of a sulcus, about $4 \mathrm{~cm}$ beneath the scalp. The opposite wall is spread apart to display the isocontours of the image on both walls. For a noiseless measurement (c), the image provides an accurate location for the source (indicated by the cross). However, because the sulcal walls are only $3 \mathrm{~mm}$ apart, the opposite
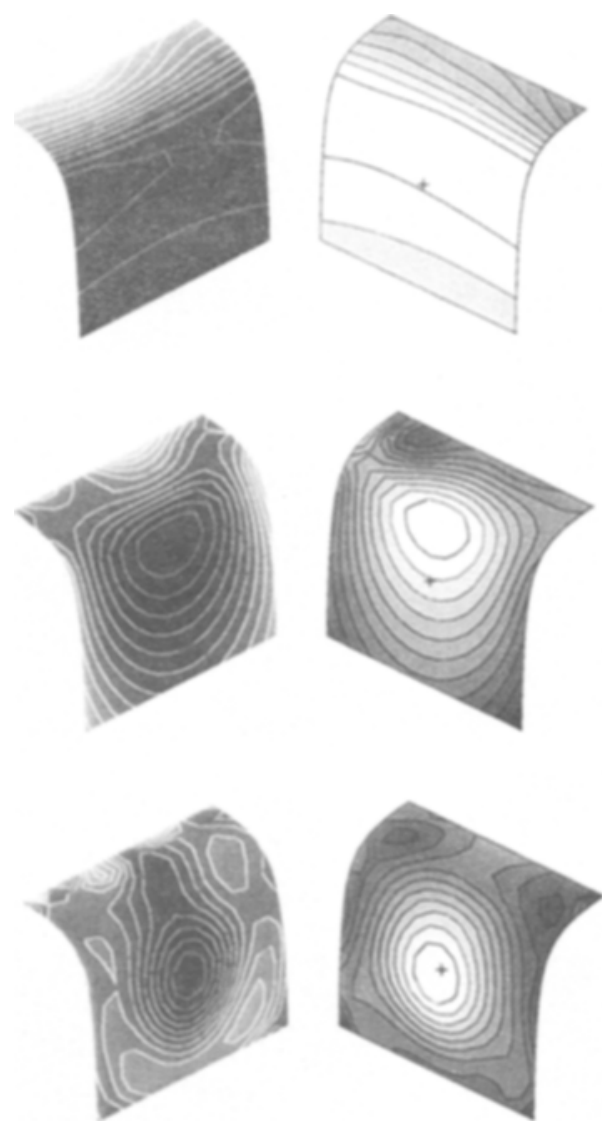

Figure 2. Magnetic source image for a current dipole located at the cross on the right wall of a sulcus, with current directed outward from the cortex. Isocontours depict the strength of the computed image for various signal-to-noise ratios, from the top: $20 \mathrm{~dB}, 40 \mathrm{~dB}$, and no noise. The opposing wall of the sulcus also displays an image, with its current directed into the cortex.

wall also displays an image, whose current has the same direction as the source but is slightly weaker. However, when the $\mathrm{S} / \mathrm{N}$ is reduced to $40 \mathrm{~dB}$, the image lies significantly higher than the source, as does the image on the opposite wall. Lateral resolution of the image is lost if $\mathrm{S} / \mathrm{N}$ is only $20 \mathrm{~dB}(\mathrm{a})$. There is a clear indication of neural activity, with some evidence of depth resolution, but the image is not of acceptable quality.

The reason why noise causes the image to lie higher than the actual sources can easily be understood. The minimum-norm least-square fitting algorithm seeks to minimize the "least square" error between the measured magnetic field and the magnetic field produced by the image; at the same time, it tries to minimize the total strength of the image. A weaker image can account for the strength of the measured field pattern if it lies closer to the magnetic field sensors, with relatively little penalty from the corresponding poorer fit to the measurements because of the noise. Overcoming this deficiency is one of the major goals of contemporary research on inverse problems. 


\section{COMPUTATIONAL REQUIREMENTS}

The computer memory that is required to compute the SVD for a realistic cortical surface for either magnetic or electric data is about $20 \mathrm{MB}$, and the computational time is $1 \mathrm{~h}$ on a Sun SPARCstation 2 when 200 magnetic field sensors or scalp electrodes are used to obtain the data. The computation time is $4 \mathrm{~h}$ for the SVD of a $400 \times 10,000$ matrix, including both magnetic and electric data, with a memory requirement of about $40 \mathrm{MB}$ when single precision is used. The execution rate of a Sun SPARCstation 2 was found to be $4 \mathrm{Mflops/sec} \mathrm{for} \mathrm{solving} \mathrm{a} \mathrm{system}$ of equations of order 100 in a FORTRAN environment using the LINPACK benchmark. By comparison, for the same study the performance of an SGI Challenge with one processor was $101 \mathrm{Mflops} / \mathrm{sec}$; and with four processors it was $998 \mathrm{Mflops} / \mathrm{sec}$ for a larger problem whose matrix was of order 1,000 , when the algorithm was modified to achieve as high an execution rate as possible. Consequently, computation time will be reduced appreciably if a high-performance computer is utilized. In addition, once the SVD of the matrices is computed for a given cortical surface and sensor/electrode array, images for different data sets can be reconstructed by simple vector multiplications. These considerations will be important to accelerate the initial steps of the solution for applications in clinical assessments of cognition and perception. However, once the SVD of the matrices is computed for a certain cortical surface and sensor/electrode array, images for different data sets can be reconstructed by simple vector multiplications.

In such a case, the condition number of matrix $\mathbf{A}$, that is, the ratio of maximum to minimum singular values, and the noise level in the measurements become important. The second term may dominate the first term because of the small singular values. The noise level in the measurements must be sufficiently small to compensate for the effects of the smallest singular values. For cases where this is not possible, the effects of the second term can be decreased by using the method of the truncated pseudoinverse. This method is simply carried out by including fewer terms in the expansion of the pseudoinverse matrix. However, although this method reduces the effects of measurement noise on the reconstructed images, it also imposes a severe loss of information because some important image components defined by higher spatial frequencies are excluded.

\section{SUPPRESSION OF ALPHA RHYTHM}

A ubiquitous feature of the normal brain is the generation of spontaneous rhythmic activity. The alpha rhythm, found in the spectral region between 8 and $14 \mathrm{~Hz}$, is the best known and often represents the strongest signal that can be recorded at the scalp. There is abundant evidence that alpha rhythm is locally suppressed (desynchronized) in cortical areas that become active, as when responding to a sensory stimulus (Berger, 1930; Kaufman \& Locker,
1970 ) or voluntarily becoming engaged in mechanical activity (Pfurtscheller \& Aranibar, 1979). Kaufman, Schwartz, Salustri, and Williamson (1990) extended the range of accessible phenomena when they observed changes in the level of alpha power recorded magnetically during performance of mental imagery and verbal tasks (Kaufman, Curtis, Wang, \& Williamson, 1992). An example of the former is a study of mental rotation (Michel, Kaufman, \& Williamson, 1994), using a paradigm introduced by Cooper and Shepard (1973).

Figure 3 illustrates the time sequence for the mental imagery experiment. A target is displayed within the interval $\mathrm{T} l$ and then a test figure is briefly displayed at the time origin. The test figure may be rotated from its original orientation (by $150^{\circ}$ or $210^{\circ}$ ), which makes the comparison more difficult. This is manifested by a longer duration of suppression, indicated by the interval designated by the line $\mathrm{S} 2$, and a correspondingly prolonged reaction time (RT) for making the decision. The duration of suppression tracks very nicely the subject's RT, indicating that the increased RT for the difficult orientation is indeed due to the need for prolonged neural processing of the mental image.

Indeed, measurements of the alpha suppression pattern across the occipital area of the head provide evidence that the occipital cortex supports the comparison required for accurate performance. When the test figure is presented as its mirror image, the RT is increased and there is a lateral shift of the magnetic field pattern in the parietal region, suggesting that more neural resources are called into play. This dynamic feature suggests that the fine temporal resolution provided by magnetic source imag-

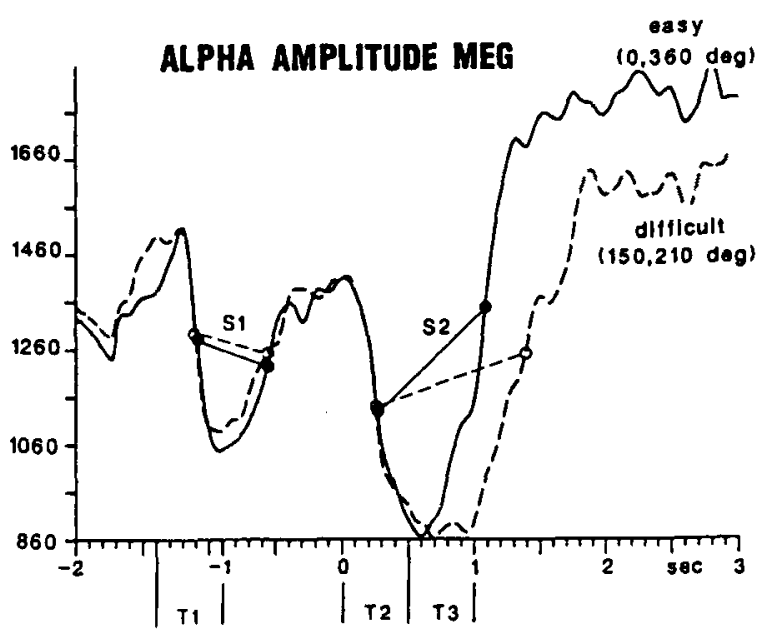

Figure 3. Alpha amplitude traces from occipital cortical activity, averaged over 30 magnetic recording positions, when a subject is presented with a memory figure during the interval $\mathrm{S} 1$ and then the same figure rotated with respect to the first is briefly presented at the time origin. The definitions for the onset and offset of alpha suppression are indicated by the closed and open circles, for both the easy and difficult orientations, respectively. The vertical scale is the mean alpha power in units of femtotesla squared. 
(a)

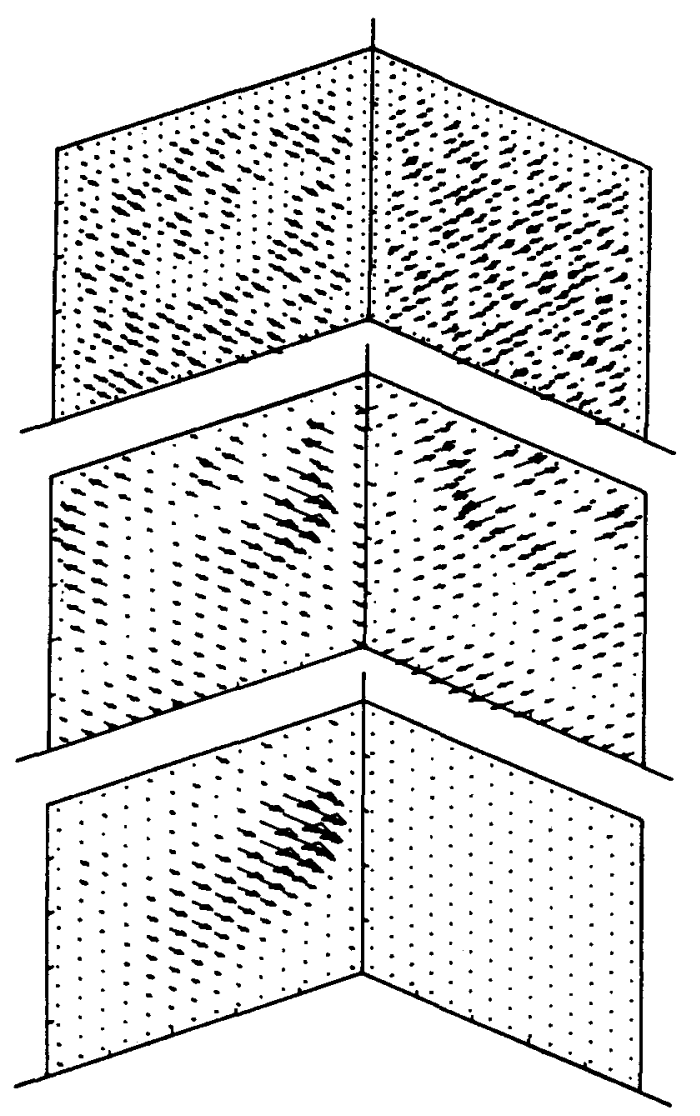

Figure 4. (a) Spontaneous alpha rhythm at a moment of time, represented on two walls of a sulcus that join at a bend. Alpha rhythm is suppressed within a circular area of the left wall. (b, c) Image power distribution across the sulcal walls obtained by subtracting the average of 100 such samples of suppressed activity from the inverse of $\mathbf{1 0 0}$ different samples of uniform activity across the wall, beginning with different seeds.

ing offers new capabilities that will be important in characterizing the working of the human brain.

Studies utilizing PET (Kosslyn et al., 1993; Roland \& Friberg, 1985) and single photon emission (SPECT) techniques (Goldenberg et al., 1989) have provided clear evidence that visual cortex supports mental arithmetic and other imagery tasks. However, these techniques do not provide fine temporal resolution.

As a consequence, Wang et al. (1993) have developed an extension of the minimum-norm least-squares estimate to provide MSIs from magnetic field power data. To determine the image across the cortex where the sources of alpha rhythm field power are attenuated, an image is first computed for the distribution of cortical current power when there is no attenuation (the baseline image). Then a new image is computed when the magnetic field power exhibits attenuation. This last image is subtracted from the baseline image to reveal the distribution of attenuated cortical currents. In this way, a temporal resolution of about $0.2 \mathrm{sec}$ can be achieved. Figure 4 illustrates this procedure for a simple cortical geometry.

This example suggests how this procedure could be realized when applied to the distribution of neural activity across the convoluted topography of the cerebral cortex. With such a procedure, we may expect that the temporal evolution of cortical activity may reveal dynamical aspects of cognitive functions that have yet to be appreciated. However, the full realization of these expectations will depend on considerable research of a mathematical nature, particularly to enhance the accuracy in establishing the depth profile of these magnetic source images.

\section{REFERENCES}

ALBERT, A. (1972). Regression and the Moore-Penrose pseudoinverse. New York: Academic Press.

Berger, H. (1930). Über das Elektrenkephalogram des Menschen II. Journal of Psychology \& Neurology, 40, 160-179.

CoOper, L. A., \& Shepard, R. N. (1973). The time required to prepare for a rotated stimulus. Memory \& Cognition, 1, 246-250.

DALE, A. M., \& SERENo, M. I. (1993). Improved localization of cortical activity by combining EEG and MEG with MRI cortical surface reconstruction. Journal of Cognitive Neuroscience, 5, 162-176.

Gençer, N. G., \& Williamson, S. J. (in press). Differential characterization of neural sources with the bimodal truncated SVD pseudoinverse for EEG and MEG measurements. IEEE Transactions on Biomedical Engineering.

George, J. S., Lewis, P. S., Ranken, D. M., Kaplan, L., \& Wood, C. C. (1991). Anatomical constraints for neuromagnetic source models. Society of Photo-Optical Instrumentation Engineers, 1443, 37-51.

Geselowitz, D. B., \& MilleR,W. T., III (1973). Extracorporeal magnetic fields generated by internal bioelectric sources. IEEE Transactions on Magnetics, 9, 392-398.

Goldenberg, G., Podreka, I, Steiner, M., Willmes, K., Suess, E., \& DEECKE, L. (1989). Regional cerebral blood flow patterns in visual imagery. Neuropsychologia, 27, 641-664.

GOLUB, G. H., \& VAN LOAN, C. F. (1989). Matrix computations (2nd ed.). Baltimore: Johns Hopkins University Press.

GuÉZIEC, A., \& DEAN, D. (1994). The wrapper: A surface optimization algorithm that preserves highly curved surfaces. In R. A. Robb (Ed.), Visualization in biomedical computing (pp. 631-642). Bellingham, WA: Society of Photo-Optical Instrumentation Engineers, International Society for Optical Engineering.

Hughett, P. (1995). An optimal constrained linear inverse method for magnetic source imaging. Annals of Biomedical Engineering, 23, 506-523.

JACKSON, D. D. (1972). Interpretation of inaccurate, insufficient and inconsistent data. Geophysical Journal of the Royal Astronomical Society, 28, 97-109.

JEFFs, B., LEAHY, R., \& SiNGH, M. (1987). An evaluation of methods for neuromagnetic image reconstruction. IEEE Transactions on Biomedical Engineering, BME-34, 713-723.

Kaufman, L., Curtis, S., Wang, J.-Z., \& Williamson, S. J. (1992). Changes in cortical activity when subjects scan memory for tones. Electroencephalography \& Clinical Neurophysiology, 82, 266-284.

Kaufman, L., \& LockER, Y. (1970). Sensory modulation of the EEG. In Proceedings of the American Psychology Association, pp. 179 180.

Kaufman, L., Schwartz, B., Salustri, C., \& Williamson, S. J. (1990). Modulation of spontaneous brain activity during mental imagery. Journal of Cognitive Neuroscience, 2, 124-132.

Kosslyn, S. M., Allpert, N. M., Thompson, W. L., Maljkovic, V., Weise, S. B., Chabris, C. F., Hamilton, S. E., Rauch, S. L., \& Buo- 
NANNO, F. S. (1993). Visual mental imagery activates topographically organized visual cortex: PET investigations. Journal of Cognitive Neuroscience, 5, 263-287.

Michel, C. M., Kaufman, L., \& Williamson, S. J. (1994). Duration of EEG and MEG $\alpha$ suppression increases with angle in a mental rotation task. Journal of Cognitive Neuroscience, 6, 139-150.

MOORE, E. H. (1920). On the reciprocal of the general algebraic matrix. Bulletin of the American Mathematical Society, 26, 394-395.

Penrose, P. (1955). A generalized inverse for matrices. Proceedings of the Cambridge Philosophical Society, 51, 406-413.

Pfurtscheller, G., \& Aranibar, A. (1979). Evaluation of eventrelated desynchronization (ERD) preceding and following voluntary self-paced movement. Electroencephalography \& Clinical Neurophysiology, 46, 138-146.

Ramon, C., Meyer, M. G., Nelson, A. C., Spelman, F. A., \& Lamp-
ING, J. (1993). Simulation studies of biomagnetic computed tomography. IEEE Transactions on Biomedical Engineering, 40, 317-322. RoLAND, P. E., \& Friberg, L. (1985). Localization of cortical areas activated by thinking. Journal of Neurophysiology, 53, 1219-1243. SHIM, Y. S., \& CHO, J. H. (1981). SVD pseudoinversion image reconstruction. IEEE Transactions on Biomedical Engineering, 29, 904909.

WANG, J.-Z., Kaufman, L., \& Williamson, S. J. (1993). Imaging regional changes in the spontaneous activity of the brain: An extension of the minimum-norm least-squares estimate. Electroencephalography \& Clinical Neurophysiology, 86, 36-50.

WANG, J.-Z., Williamson, S. J., \& KaufMan, L. (1992). Magnetic source images determined by a lead-field analysis: The unique minimum-norm least-squares estimation. IEEE Transactions on Biomedical Engineering, 39, 665-675. 